\title{
Multi-soliton dynamics of anti-self-dual gauge fields
}

\author{
Masashi Hamanaka and Shan-Chi Huang \\ Department of Mathematics, Nagoya University, \\ Nagoya, 464-8602, Japan \\ E-mail: hamanaka@math.nagoya-u.ac.jp, x18003x@math.nagoya-u.ac.jp
}

ABSTRACT: We study dynamics of multi-soliton solutions of anti-self-dual Yang-Mills equations for $G=\mathrm{GL}(2, \mathbb{C})$ in four-dimensional spaces. The one-soliton solution can be interpreted as a codimension-one soliton in four-dimensional spaces because the principal peak of action density localizes on a three-dimensional hyperplane. We call it the soliton wall. We prove that in the asymptotic region, the $n$-soliton solution possesses $n$ isolated localized lumps of action density, and interpret it as $n$ intersecting soliton walls. More precisely, each action density lump is essentially the same as a soliton wall because it preserves its shape and "velocity" except for a position shift of principal peak in the scattering process. The position shift results from the nonlinear interactions of the multi-solitons and is called the phase shift. We calculate the phase shift factors explicitly and find that the action densities can be real-valued in three kind of signatures. Finally, we show that the gauge group can be $G=\mathrm{SU}(2)$ in the Ultrahyperbolic space $\mathbb{U}$ (the split signature $(+,+,-,-)$ ). This implies that the intersecting soliton walls could be realized in all region in $\mathrm{N}=2$ string theories. It is remarkable that quasideterminants dramatically simplify the calculations and proofs.

KEywords: Integrable Field Theories, Solitons Monopoles and Instantons, Duality in Gauge Field Theories, Integrable Hierarchies

ARXiv EPrint: 2106.01353 


\section{Contents}

1 Introduction 1

2 Brief introduction to quasideterminants 3

$\begin{array}{lll}3 & \text { ASDYM equations and quasi-Wronskian solutions } & 6\end{array}$

4 Multi-soliton solutions of ASDYM equation $\quad 7$

$\begin{array}{lll}4.1 & \text { One-soliton solutions of ASDYM equation } & 8\end{array}$

$\begin{array}{lll}4.2 & \text { Asymptotic behavior of the } n \text {-soliton solutions } & 9\end{array}$

5 Unitarity of the multi-soliton solutions in $\mathbb{U} \quad 14$

6 Conclusion and discussion $\quad 14$

\section{Introduction}

Soliton theories and integrable systems have been studied actively and developed rapidly in the past sixty years. Meanwhile, the applications of exact solitons, such as instantons, monopoles, vortices, and domain walls also promote the developments in different fields of mathematics and theoretical physics. Therefore, systematic construction of exact soliton solutions has been one of the most attractive topic in the studies of integrable systems. Stability of multi-soliton solutions closely relates to the existence of infinite many conserved quantities which leads to an infinite dimensional symmetry of the integrable systems. Among these studies, Sato's theory of solitons is one of the most appealing result which reveals an infinite dimensional symmetry behind the KP equation and gives a comprehensive viewpoint to unify the theory of lower-dimensional integrable systems [39, 40]. The key ingredients of Sato's theory are integrable hierarchies and tau functions. The tau functions can be represented specifically as Wronskian determinants. This fact is crucial to demonstrate that Hirota bilinear equations are just Plücker relations by using Maya diagram representations [38-40]. Combining them with the integrable hierarchies, we have infinite Plücker relations which define an infinite dimensional Grassmann manifold as the solution space of the KP equation. In this way, the infinite dimensional symmetry is clarified.

In four-dimensional integrable systems, the most beautiful one would be the antiself-dual Yang-Mills (ASDYM) equations. The essence of hidden integrability behind the anti-self-dual Yang-Mills equations can be captured clearly under the description of the twistor theory (e.g. [5, 27, 34, 35, 43]). On the other hand, the anti-self-dual Yang-Mills equations can be reduced to various lower-dimensional soliton equations, such as the KdV equation and the nonlinear Schrödinger equation, by suitable reduction procedures [27, 42]. This fact suggests that there might be a profound connection between the Sato's theory 
and the twistor theory, which perhaps leads to higher-dimensional extension of Sato's theory. A feasible approach to anti-self-dual Yang-Mills equations from the viewpoint of Sato's theory is mentioned in [41], while the description of tau-functions is still unclarified. Another remarkable result is from the viewpoint of Bäcklund transformations [2]. This kind of exact solutions can be represented by determinants in a regular pattern, however, not the Wronskian type determinants. Therefore, the description of tau-functions remains to be clarified. Furthermore, several attempts were made by us to construct one-soliton solutions from [2], and the resulting action density is $\operatorname{Tr} F_{\mu \nu} F^{\mu \nu}=0$ [19]. Perhaps for this reason, only few discussions have been made (as far as the authors know) in this direction for a long time (e.g. [1, 3, 26]).

Just last year, we had made some progress in this direction. More precisely, we constructed Wronskian type solutions of the anti-self-dual Yang-Mills equations successfully by applying a Darboux transformation [29] in the noncommutative (NC) framework [10]. This Wronskian type solutions can be represented in terms of quasideterminants [9] (called the quasi-Wronskian solutions, for short). A highly nontrivial result of quasi-Wronskian solutions is that the action density in one-soliton case is no longer zero [19]. Moreover, the principal peak of the action density lies on a three-dimensional hyperplane. Therefore, our solutions can be interpreted as codimension-one solitons in four-dimensional space. We call them the soliton walls to distinguish them from the domain walls in this paper. Now a natural question comes: can we find the behavior of $n$ intersecting soliton walls from the quasi-Wronskian solution? The answer is yes and we verify this by analyzing the asymptotic behavior of $n$-soliton solution (4.7) in the scattering process. This guarantees the stability and integrability of the intersecting soliton walls like KP multi-solitons. Moreover, the quasi-Wronskian descriptions are actually more essential than the ordinary Wronskian. This would lead to analogs of the Maya-diagram representation, tau-functions, and perhaps Sato's formulations of the anti-self-dual Yang-Mills equations.

In this paper, we clarify the asymptotic dynamics of multi-soliton solutions of anti-selfdual Yang-Mills equations for $G=\mathrm{GL}(2, \mathbb{C})$ in four-dimensional spaces. We prove that in the asymptotic region, the $n$-soliton solution possesses $n$ isolated localized lumps of action density, and interpret it as $n$ intersecting soliton walls. More precisely, each action density lump is essentially the same as a soliton wall because it preserves the shape and "velocity" except for a phase shifts. Furthermore, we show that in the Ultrahyperbolic space $\mathbb{U}$, the gauge group can be $\mathrm{U}(2)$. This result is important for physical interpretations because the anti-self-dual Yang-Mills equations in this space are equations of motion of effective actions for open $\mathrm{N}=2$ string theories [13, 25, 31, 32]. Therefore, the intersecting soliton walls could be realized in the $\mathrm{N}=2$ string theory in all region of the space-time as new physical objects, that is, intersecting branes. It is remarkable that the quasideterminants play crucial roles in calculations and proofs. As we will comment in the end of section 2 and in the beginning of subsection 4.2, matrix elements of our quasi-Wronskian solutions consist of $2 \times 2$ matrices rather than scalar functions. This "non-abelian treatment" is quite important to make all proofs and discussions drastically simple and expected to be applied to various non-abelian integrable systems. 
This paper is organized as follows. In section 2, we make a brief introduction to quasideterminants and summarize some properties of them. These are useful mathematical tools for later sections. In section 3, we introduce the $J$-matrix formulation [27] of antiself-dual Yang-Mills equations and the quasi-Wronskian solution [10]. In subsection 4.1, we review exact one-soliton solutions and the interpretation of soliton walls. In subsection 4.2 , we study the asymptotic behavior of multi-soliton solution and give the interpretation of intersecting soliton walls. In section 5, we prove that the intersecting soliton walls can be embedded into $G=\mathrm{SU}(2)$ gauge theory on the Ultrahyperbolic space by showing that gauge fields are all anti-hermitian. Section 6 is devoted to conclusion and discussion.

\section{Brief introduction to quasideterminants}

In this section, we give a brief introduction to quasideterminants defined firstly by Gelfand and Retakh [9]. For detailed discussion, see e.g. [8, 21]. Briefly speaking, the quasideterminant of a $n \times n$ matrix $\mathrm{X}$ is a noncommutative generalization of the ratio of the determinant of $\mathrm{X}$ to the determinant of a $(n-1) \times(n-1)$ submatrix. Therefore, quasideterminant is related to the inverse matrix of $X$. Here we assume the existence of the invertible matrix $X$.

Let $X=\left(x_{i j}\right)$ be a $n \times n$ invertible matrix over a noncommutative ring and $Y=\left(y_{i j}\right)$ be the inverse matrix of $X: X Y=Y X=1$. Then the $(i, j)$-th quasideterminant of $X$ is defined as the inverse of an element of $Y=X^{-1}$ :

$$
|X|_{i j}:=y_{j i}^{-1}
$$

A convenient representation for $(i, j)$-th quasideterminant is

$$
|X|_{i j}=\left|\begin{array}{ccccc}
x_{11} & \cdots & x_{1 j} & \cdots & x_{1 n} \\
\vdots & & \vdots & & \vdots \\
x_{i 1} & \cdots & x_{i j} & \cdots & x_{i n} \\
\vdots & & \vdots & & \vdots \\
x_{n 1} & \cdots & x_{n j} & \cdots & x_{n n}
\end{array}\right| .
$$

To expand (2.2), let us introduce the inverse matrix formula for $2 \times 2$ block matrix:

$$
\left(\begin{array}{ll}
A & B \\
C & d
\end{array}\right)^{-1}=\left(\begin{array}{cc}
A^{-1}+A^{-1} B S^{-1} C A^{-1} & -A^{-1} B S^{-1} \\
-S^{-1} C A^{-1} & S^{-1}
\end{array}\right)
$$

where $A$ is a square matrix, $d$ is a single element and $S:=d-C A^{-1} B$ is called the Schur complement. We note that any invertible matrix can be decomposed into a $2 \times 2$ block matrix and one of the diagonal terms is size $1 \times 1$. We can assign $A$ to be $X^{i j}, B$ to be $X^{i}{ }_{j}, C$ to be $X_{i}{ }^{j}$, and $d$ to be $x_{i j}$, where $X^{i j}$ denotes the submatrix obtained from $X$ by deleting $i$-th row and $j$-th column, $X^{i}{ }_{j}$ and $X_{i}{ }^{j}$ denote the submatrices obtained from $j$-th column and $i$-th row of $X$ by deleting $x_{i j}$, respectively. Now the $(i, j)$-th quasideterminant 
can be expressed as the Schur complement:

$$
\begin{aligned}
|X|_{i j} & =S=x_{i j}-X_{i}^{j}\left(X^{i j}\right)^{-1} X_{j}^{i} \\
& =x_{i j}-\sum_{i^{\prime}(\neq i), j^{\prime}(\neq j)} x_{i i^{\prime}}\left(\left|X^{i j}\right|_{j^{\prime} i^{\prime}}\right)^{-1} x_{j^{\prime} j},
\end{aligned}
$$

By using this, explicit representations of the quasideterminants can be obtained iteratively. For example, for a $1 \times 1$ matrix $X=x$

$$
|X|=x
$$

and for a $2 \times 2$ matrix $X=\left(x_{i j}\right)$

$$
\begin{array}{rlrl}
|X|_{11} & =\left|\begin{array}{ll}
x_{11} & x_{12} \\
x_{21} & x_{22}
\end{array}\right|=x_{11}-x_{12} x_{22}^{-1} x_{21}, & |X|_{12}=\left|\begin{array}{ll}
x_{11} & x_{12} \\
x_{21} & x_{22}
\end{array}\right|=x_{12}-x_{11} x_{21}^{-1} x_{22}, \\
|X|_{21}=\left|\begin{array}{ll}
x_{11} & x_{12} \\
x_{21} & x_{22}
\end{array}\right|=x_{21}-x_{22} x_{12}^{-1} x_{11}, & |X|_{22}=\left|\begin{array}{ll}
x_{11} & x_{12} \\
x_{21} & x_{22}
\end{array}\right|=x_{22}-x_{21} x_{11}^{-1} x_{12},
\end{array}
$$

and for a $3 \times 3$ matrix $X=\left(x_{i j}\right)$

$$
\begin{aligned}
& |X|_{11}=\left|\begin{array}{lll}
x_{11} & x_{12} & x_{13} \\
x_{21} & x_{22} & x_{23} \\
x_{31} & x_{32} & x_{33}
\end{array}\right|=x_{11}-\left(x_{12}, x_{13}\right)\left(\begin{array}{ll}
x_{22} & x_{23} \\
x_{32} & x_{33}
\end{array}\right)^{-1}\left(\begin{array}{l}
x_{21} \\
x_{31}
\end{array}\right)
\end{aligned}
$$

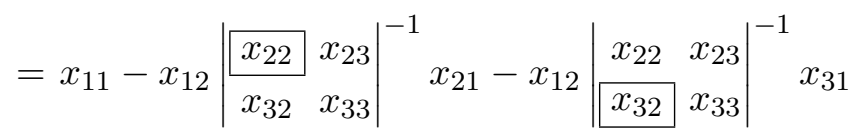

$$
\begin{aligned}
& -x_{13}\left|\begin{array}{ll}
x_{22} & x_{23} \\
x_{32} & x_{33}
\end{array}\right|^{-1} x_{21}-x_{13}\left|\begin{array}{ll}
x_{22} & x_{23} \\
x_{32} & x_{33}
\end{array}\right|^{-1} x_{31},
\end{aligned}
$$

and so on. We remark that the following expressions of quasideterminants are exactly the same because the Schur complement are all equivalent.

$$
\left|\begin{array}{ll}
A & B \\
C & d
\end{array}\right|=\left|\begin{array}{ll}
C & d \\
A & B
\end{array}\right|=\left|\begin{array}{ll}
d & C \\
B & A
\end{array}\right|=\left|\begin{array}{cc}
B & A \\
d & C
\end{array}\right|=d-C A^{-1} B .
$$

Now let us introduce some important properties and identities of quasideterminants, which are relevant to discussions in this paper.

Proposition 1 ([8, 9, 21]). Let $A=\left(a_{i j}\right)$ be a square matrix of order $n$ in (i) $\sim($ iii $)$, while in (iv) and (v), appropriate partitions are made so that all matrices in quasideterminants are square.

(i) Permutation of rows and columns

The quasideterminant $|A|_{i j}$ does not depend on permutations of rows and columns in the matrix A. 
(ii) The common multiplication of rows and columns

For any invertible elements $\Lambda_{j}(j=1, \cdots, n)$, we have

$$
\left|\begin{array}{ccccc}
a_{1,1} \Lambda_{1} & \cdots & a_{1, j} \Lambda_{j} & \cdots & a_{1, n} \Lambda_{n} \\
\vdots & & \vdots & & \vdots \\
a_{i, 1} \Lambda_{1} & \cdots & a_{i, j} \Lambda_{j} & \cdots & a_{i, n} \Lambda_{n} \\
\vdots & & \vdots & & \vdots \\
a_{n, 1} \Lambda_{1} & \cdots & a_{n, j} \Lambda_{j} & \cdots & a_{n, n} \Lambda_{n}
\end{array}\right|=\left|\begin{array}{ccccc}
a_{1,1} & \cdots & a_{1, j} & \cdots & a_{1, n} \\
\vdots & & \vdots & & \vdots \\
a_{i, 1} & \cdots & a_{i, j} & \cdots & a_{i, n} \\
\vdots & & \vdots & & \vdots \\
a_{n, 1} & \cdots & a_{n, j} & \cdots & a_{n, n}
\end{array}\right| \Lambda_{j} .
$$

We note that in the left hand side of (2.5), the common elements $\Lambda_{j}$ must appear in the right side of the same column. On the other hand, if the common elements appear in the left side of the same row, one can get the rule for common multiplication of rows.

(iii) The addition of rows and columns

Let the matrix $N=\left(n_{i j}\right)$ be obtained from the matrix $A$ by replacing the $k$-th column of $A$ with the sum of the $k$-th column and l-th column, that is, $n_{i k}=a_{i k}+a_{i l}$ and $n_{i j}=a_{i j}$ for $k \neq j$. Then

$$
|A|_{i j}=|N|_{i j}, \quad \text { for } j \neq k
$$

(The addition of rows is similar).

(iv) Noncommutative Jacobi identity [12] (An useful and simplified version of the noncommutative Sylvester's Theorem [9]):

$$
\left|\begin{array}{ccc}
a & R & b \\
P & M & Q \\
c & S & d
\end{array}\right|=\left|\begin{array}{cc}
M & Q \\
S & d
\end{array}\right|-\left|\begin{array}{cc}
P & M \\
c & S
\end{array}\right|\left|\begin{array}{ll}
a & R \\
P & M
\end{array}\right|^{-1}\left|\begin{array}{cc}
R & b \\
M & Q
\end{array}\right| .
$$

(v) Homological relations [9, 12]

$$
\left|\begin{array}{ccc}
a & R & b \\
P & M & Q \\
c & S & d
\end{array}\right|=\left|\begin{array}{ccc}
a & R & b \\
P & M & Q \\
c & S & d
\end{array}\right|\left|\begin{array}{ccc}
a & R & b \\
P & M & Q \\
0 & 0 & 1
\end{array}\right|, \quad\left|\begin{array}{ccc}
a & R & b \\
P & M & Q \\
c & S & d
\end{array}\right|=\left|\begin{array}{ccc}
a & R & 0 \\
P & M & 0 \\
c & S & 1
\end{array}\right|\left|\begin{array}{ccc}
a & R & b \\
P & M & Q \\
c & S & d
\end{array}\right| .
$$

If we use the homological relation again on the right hand side, we can obtain the following inverse relation immediately:

$$
\left|\begin{array}{ccc}
a & R & b \\
P & M & Q \\
0 & 0 & 1
\end{array}\right|^{-1}=\left|\begin{array}{ccc}
a & R & b \\
P & M & Q \\
1 & 0 & 0
\end{array}\right|, \quad\left|\begin{array}{ccc}
a & R & 0 \\
P & M & 0 \\
c & S & 1
\end{array}\right|^{-1}=\left|\begin{array}{ccc}
a & R & 1 \\
P & M & 0 \\
c & S & 0
\end{array}\right| .
$$

We note that the definition of the quasideterminants and proposition 1 are valid even if the matrix elements belong to noncommutative associative algebras. This means that we can consider all elements $x_{i j}$ in (2.2) as $N \times N$ matrices (in this case, $X$ is $n N \times n N$ ). 
For our purpose in this paper, we will just consider the case of $2 \times 2$ matrices because the gauge group is $G=G L(2, \mathbb{C})$. In this case, we can expand the quasideterminant as a $2 \times 2$ matrix and the matrix elements are four quasideterminants.

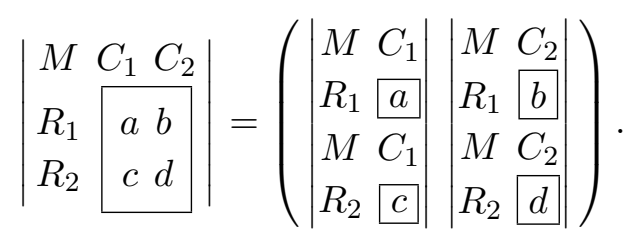

Note that in the right hand side, each box includes a $1 \times 1$ scalar element. Hence they can be represented as a ratio of ordinary determinants by virtue of the Laplace formula on inverse matrices:

$$
|X|_{i j}=y_{j i}^{-1}=(-1)^{i+j} \frac{\operatorname{det} X}{\operatorname{det} X^{i j}}
$$

where $X^{i j}$ is a matrix obtained from $X$ by deleting $i$-th row and $j$-th column.

\section{ASDYM equations and quasi-Wronskian solutions}

In this section, we review anti-self-dual Yang-Mills equations in four-dimensional flat spaces whose real coordinates are denoted by $x^{\mu}(\mu=0,1,2,3)$. To facilitate the discussion, we set the gauge group to be $G=\mathrm{GL}(N, \mathbb{C})$ (or subgroup of $\mathrm{GL}(N, \mathbb{C})$ ).

Firstly, let us consider a four-dimensional complex space with coordinates $(z, \widetilde{z}, w, \widetilde{w})$ and define the metric to be $d s^{2}=2(d z d \widetilde{z}-d w d \widetilde{w})$. We can recover various real spaces from this complex space by imposing suitable conditions on $z, \widetilde{z}, w, \widetilde{w}$. We call them the real slice conditions in this paper. For example, the Euclidean real space $\mathbb{E}$ is given by $\widetilde{z}=\bar{z}, \widetilde{w}=$ $-\bar{w}$, the Minkowski real space $\mathbb{M}$ is by $z, \widetilde{z} \in \mathbb{R}, \widetilde{w}=\bar{w}$, and the Ultrahyperbolic real space $\mathbb{U}$ is by $z, \widetilde{z}, w, \widetilde{w} \in \mathbb{R}$. Explicit relations between $z, \widetilde{z}, w, \widetilde{w}$ and $x^{\mu}$ are summarized in table 1 in subsection 4.1 .

Now we introduce an equivalent representation of the anti-self-dual Yang-Mills equations on this complex space, called Yang's equation:

$$
\partial_{\widetilde{z}}\left(\partial_{z} J \cdot J^{-1}\right)-\partial_{\widetilde{w}}\left(\partial_{w} J \cdot J^{-1}\right)=0,
$$

where $J$ is an $N \times N$ complex matrix. This formulation gives a more concise way to unify anti-self-dual Yang-Mills equations of various real spaces to one complex form and extensively used in the field of integrable systems. Moreover, the anti-self-dual gauge fields can be expressed explicitly by the solution $J$ of Yang's equation in a convenient gauge:

$$
A_{z}=-\partial_{z} J \cdot J^{-1}, \quad A_{w}=-\partial_{w} J \cdot J^{-1}, \quad A_{\widetilde{z}}=A_{\widetilde{w}}=0,
$$

We can easily to check that (3.2) actually satisfies the anti-self-dual Yang-Mills equation of a complex representation:

$$
F_{z w}=0, \quad F_{\tilde{z} \widetilde{w}}=0, \quad F_{z \widetilde{z}}-F_{w \widetilde{w}}=0,
$$


where $F_{z w}:=\partial_{z} A_{w}-\partial_{w} A_{z}+\left[A_{z}, A_{w}\right]$ and so on denote the field strengths. By taking real slice conditions on $z, \widetilde{z}, w, \widetilde{w}$ as mentioned above, (3.3) reduces to the standard anti-selfdual Yang-Mills equations in four-dimensional real spaces in the sense of the Hodge dual operator.

For the sake of generating exact solutions systematically, a typical technique developed in the field of integrable systems is to find the Lax representations, and then use the covariance of Lax equations under the Darboux transformation to generate more exact solutions. Here we introduce a formulation, slightly different from the conventional Lax formalism. Let us consider the following linear system [29]:

$$
\begin{aligned}
L(\phi) & :=J \partial_{w}\left(J^{-1} \phi\right)-\left(\partial_{\widetilde{z}} \phi\right) \zeta=0, \\
M(\phi) & :=J \partial_{z}\left(J^{-1} \phi\right)-\left(\partial_{\widetilde{w}} \phi\right) \zeta=0,
\end{aligned}
$$

where $\zeta$ is a matrix generalization of the spectral parameter. More precisely, $\zeta$ is an $N \times N$ constant matrix. We can show that the compatibility condition $L(M(\phi))-M(L(\phi))=0$ implies Yang's equation (3.1) and the linear system (3.4) is covariant under the following Darboux transformation [29]:

$$
\widetilde{\phi}=\phi \zeta-\theta \Lambda \theta^{-1} \phi, \quad \widetilde{J}=-\theta \Lambda \theta^{-1} J,
$$

where $\theta$ is an eigenfunction of the linear system (3.4) for the choice of eigenvalue $\zeta=\Lambda$.

After $n$ iteration of the Darboux transformation, we get an exact solution $J_{n}$ of Yang's equation from a trivial seed solution $J=1$ and this kind of solution can be expressed in terms of quasideterminant in a compact form [29]. Here we use the terminology Wronskian type quasideterminants, or quasi-Wronskian for short. The specific form of $J_{n}$ is as follows:

$$
J_{n}=\left|\begin{array}{cccc}
\theta_{1} & \cdots & \theta_{n} & 1 \\
\theta_{1} \Lambda_{1} & \cdots & \theta_{n} \Lambda_{n} & 0 \\
\vdots & & \vdots & \vdots \\
\theta_{1} \Lambda_{1}^{n-1} & \cdots & \theta_{n} \Lambda_{n}^{n-1} & 0 \\
\theta_{1} \Lambda_{1}^{n} & \cdots & \theta_{n} \Lambda_{n}^{n} & 0
\end{array}\right|,
$$

where $\left(\theta_{i}, \Lambda_{i}\right)(i=1,2, \cdots, n)$ are pairs of eigenfunctions and eigenvalues of the initial linear system for $J=1$ in (3.4) [10, 29]:

$$
\partial_{w} \theta_{i}=\left(\partial_{z} \theta_{i}\right) \Lambda_{i}, \quad \partial_{z} \theta_{i}=\left(\partial_{\widetilde{w}} \theta_{i}\right) \Lambda_{i}
$$

We remark that in $G=\operatorname{GL}(N, \mathbb{C})$ case, all the $\theta_{i}$ and $\Lambda_{i}$ are $N \times N$ matrices. Therefore, $J_{n}$ is exactly a $N \times N$ matrix if we expand it term by term as the Schur complement (2.3).

\section{Multi-soliton solutions of ASDYM equation}

As mentioned in previous section, we can obtain $n$ different pairs of $\left(\theta_{i}, \Lambda_{i}\right)$ by solving (3.7) and use them to form exact solutions $J_{n}$ of Yang's equation. One kind of $n$-soliton solutions 
for $G=\mathrm{GL}(2, \mathbb{C})$ is given by an interesting case of $J_{n}$ which is composed of a special set of solutions of (3.7) [10]:

$$
\theta_{i}=\left(\begin{array}{cc}
a_{i} e^{L_{i}} & b_{i} e^{-M_{i}} \\
c_{i} e^{-L_{i}} & d_{i} e^{M_{i}}
\end{array}\right), \quad \Lambda_{i}=\left(\begin{array}{cc}
\lambda_{i} & 0 \\
0 & \mu_{i}
\end{array}\right),
$$

where $i=1,2, \cdots, n, L_{i}=\lambda_{i} \beta_{i} z+\alpha_{i} \widetilde{z}+\lambda_{i} \alpha_{i} w+\beta_{i} \widetilde{w}, M_{i}:=\mu_{i} \delta_{i} z+\gamma_{i} \widetilde{z}+\mu_{i} \gamma_{i} w+$ $\delta_{i} \widetilde{w}$, and $\lambda_{i}, \mu_{i}, a_{i}, b_{i}, c_{i}, d_{i}, \alpha_{i}, \beta_{i}, \gamma_{i}, \delta_{i}$ are complex constants. Furthermore, we confirm that in subsection 4.2, a reduced version of solution (4.1) (cf. (4.7)) actually generates a multi-soliton distribution because this distribution has $n$ localized action densities in the asymptotic region. Hence this multi-soliton is stable in the scattering process.

\subsection{One-soliton solutions of ASDYM equation}

In this subsection we summarize some results and properties of the one-soliton solutions in our previous work [19]. The $J$-matrix is

$$
J=\left|\begin{array}{cc}
\theta & 1 \\
\theta \Lambda & 0
\end{array}\right|=-\theta \Lambda \theta^{-1}, \quad \theta=\left(\begin{array}{cc}
a e^{L} & b e^{-M} \\
c e^{-L} & d e^{M}
\end{array}\right), \quad \Lambda=\left(\begin{array}{cc}
\lambda & 0 \\
0 & \mu
\end{array}\right) .
$$

The action density of this kind of solution is calculated as follows:

$$
\begin{gathered}
\operatorname{Tr} F^{2}=8(\lambda-\mu)^{2}(\alpha \delta-\beta \gamma)^{2}\left(2 \operatorname{sech}^{2} X-3 \operatorname{sech}^{4} X\right), \\
\text { where } X:=M+L+\frac{1}{2} \log (-a d / b c) .
\end{gathered}
$$

We remark that (4.3) is a highly nontrivial result and cannot be realized in the conventional Lax formalism. More explicitly, if the spectral parameter matrix $\Lambda$ is a scalar matrix (i.e. $\lambda=\mu$ ), then linear system (3.4) is equivalent to the conventional one and the resulting action density (4.3) becomes trivial: $\operatorname{Tr} F^{2}=0$.

By imposing real slice conditions on the space-time coordinates $z, \widetilde{z}, w, \widetilde{w}$, and putting an additional condition on $J$-matrix: $d=\bar{a}, c=-\bar{b}$ and $M=\bar{L}$, we can get one-soliton solutions on different real spaces $\mathbb{E}, \mathbb{M}$ and $\mathbb{U}$ :

$$
J=\left|\begin{array}{cc}
\theta & 1 \\
\theta \Lambda & 0
\end{array}\right|=-\theta \Lambda \theta^{-1}, \quad \theta=\left(\begin{array}{cc}
a e^{L} & b e^{-\bar{L}} \\
-\bar{b} e^{-L} & \bar{a} e^{\bar{L}}
\end{array}\right), \quad \Lambda=\left(\begin{array}{cc}
\lambda & 0 \\
0 & \mu
\end{array}\right) .
$$

The resulting action densities take real value:

$$
\operatorname{Tr} F_{\mu \nu} F^{\mu \nu}=C\left(2 \operatorname{sech}^{2} X-3 \operatorname{sech}^{4} X\right)
$$

where $X:=L+\bar{L}+\log |a / b|$ and $C$ is a real constant depending on the signature of different real spaces. We note that the principal peak of these action densities lie on a three-dimensional hyperplane defined by $X=L+\bar{L}+\log |a / b|=0$ with normal vector $l_{\mu}+\bar{l}_{\mu}$, where $l_{\mu}$ is defined by $L=l_{\mu} x^{\mu}$ (cf. table 1 ). Therefore, we can interpret them as codimension-one solitons in four dimensional spaces and use the terminology soliton walls in this paper to distinguish our solution from domain walls.

Main results of [19] are summarized in table 1. 


\begin{tabular}{|c|c|c|c|}
\hline signature & $\mathbb{E}$ & $\mathbb{M}$ & $\bar{U}$ \\
\hline $\begin{array}{c}\text { real slice } \\
\sqrt{2}\left(\begin{array}{cc}\widetilde{z} & w \\
\widetilde{w} & z\end{array}\right)=\end{array}$ & $\begin{array}{c}\widetilde{z}=\bar{z}, \widetilde{w}=-\bar{w} \\
\left(\begin{array}{c}x^{0}+i x^{1}-x^{2}+i x^{3} \\
x^{2}+i x^{3} x^{0}-i x^{1}\end{array}\right)\end{array}$ & $\begin{array}{c}z, \widetilde{z} \in \mathbb{R}, \widetilde{w}=\bar{w} \\
\left(\begin{array}{cc}x^{0}+x^{1} & x^{2}-i x^{3} \\
x^{2}+i x^{3} & x^{0}-x^{1}\end{array}\right)\end{array}$ & 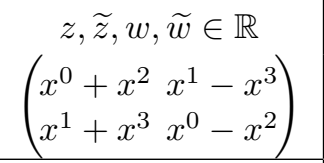 \\
\hline $\begin{array}{l}\text { reality condition } \\
\qquad L=\bar{M}\end{array}$ & $\begin{array}{c}\mu=-1 / \bar{\lambda} \\
L=(\lambda \beta) z+\alpha \bar{z} \\
+(\lambda \alpha) w-\beta \bar{w}\end{array}$ & $\begin{array}{c}\text { NONE } \\
L=(\lambda \bar{\mu} \alpha) z+\alpha \widetilde{z} \\
+(\lambda \alpha) w+(\bar{\mu} \alpha) \bar{w}\end{array}$ & $\begin{array}{c}\mu=\bar{\lambda} \\
L=(\lambda \beta) z+\alpha \widetilde{z} \\
+(\lambda \alpha) w+\beta \widetilde{w}\end{array}$ \\
\hline$L=l_{\mu} x^{\mu}$ & $l_{\mu}=\frac{1}{\sqrt{2}}\left(\begin{array}{c}\alpha+\lambda \beta \\
i(\alpha-\lambda \beta) \\
\beta-\lambda \alpha \\
i(\beta+\lambda \alpha)\end{array}\right)$ & $l_{\mu}=\frac{\alpha}{\sqrt{2}}\left(\begin{array}{c}1+\lambda \bar{\mu} \\
1-\lambda \bar{\mu} \\
\bar{\mu}+\lambda \\
i(\bar{\mu}-\lambda)\end{array}\right)$ & $l_{\mu}=\frac{1}{\sqrt{2}}\left(\begin{array}{c}\alpha+\lambda \beta \\
\beta-\lambda \alpha \\
\alpha-\lambda \beta \\
\beta+\lambda \alpha\end{array}\right)$ \\
\hline constant $C$ & $8\left(|\alpha|^{2}+|\beta|^{2}\right)^{2}\left(|\lambda|^{2}+1\right)^{2}$ & $8\left|\alpha^{2}(\lambda-\mu)\right|^{4}$ & $8[(\alpha \bar{\beta}-\bar{\alpha} \beta)(\lambda-\bar{\lambda})]^{2}$ \\
\hline $\begin{array}{l}\text { hermicity } \\
\text { of } A_{\mu}\end{array}$ & $\begin{array}{c}A_{0}, A_{2} \text { :anti-hermitian } \\
A_{1}, A_{3}: \text { hermitian } \\
\quad(\text { when } \lambda= \pm i)\end{array}$ & $\begin{array}{c}A_{0}, A_{1}, A_{2} \text { :anti-hermitian } \\
A_{3} \text { :hermitian } \\
(\text { when } \lambda=\bar{\mu})\end{array}$ & $\begin{array}{l}A_{0}, A_{1}, A_{2}, A_{3}: \\
\text { anti-hermitian } \\
\quad(\mathrm{NONE})\end{array}$ \\
\hline gauge gr & $\mathrm{G}=\mathrm{SL}(2, \mathbb{C})$ & $\mathrm{G}=\mathrm{SL}(2, \mathbb{C})$ & $\mathrm{G}=\mathrm{SU}(2)$ \\
\hline
\end{tabular}

Table 1. Summary of one-soliton solutions.

\subsection{Asymptotic behavior of the $n$-soliton solutions}

Now let us put the condition: $d_{i}=\bar{a}_{i}, c_{i}=-\bar{b}_{i}, M_{i}=\bar{L}_{i}$ on (4.1) and discuss the asymptotic behavior of the $n$-soliton solution $(i=1,2, \cdots, n)$ :

$$
J_{n}=\left|\begin{array}{cccc}
\theta_{1} & \cdots & \theta_{n} & 1 \\
\theta_{1} \Lambda_{1} & \cdots & \theta_{n} \Lambda_{n} & 0 \\
\vdots & & \vdots & \vdots \\
\theta_{1} \Lambda_{1}^{n} & \cdots & \theta_{n} \Lambda_{n}^{n} & 0
\end{array}\right|, \theta_{i}=\left(\begin{array}{cc}
a_{i} e^{L_{i}} & b_{i} e^{-\bar{L}_{i}} \\
-\bar{b}_{i} e^{-L_{i}} & \bar{a}_{i} e^{\bar{L}_{i}}
\end{array}\right), \Lambda_{i}=\left(\begin{array}{cc}
\lambda_{i} & 0 \\
0 & \mu_{i}
\end{array}\right),
$$

where $L_{i}=\lambda_{i} \beta_{i} z+\alpha_{i} \widetilde{z}+\lambda_{i} \alpha_{i} w+\beta_{i} \widetilde{w}$, and $a_{i}, b_{i}, \alpha_{i}, \beta_{i}, \lambda_{i}, \mu_{i} \in \mathbb{C}$. We assume here that $L_{i}$ $(i=1,2, \cdots, n)$ are independent with each other and there is no special relation between them. In other words, we just consider the situation of pure-soliton scattering and exclude the case of resonance processes.

Inspired by a typical technique that was used for discussing the asymptotic behavior of Wronskian type $n$-soliton solutions of the KP equation, we follow a quite similar procedure like this to deal with quasi-Wronskian type $n$-soliton solutions of the anti-self-dual YangMills equations. It is actually a new attempt because the elements $\theta_{i}, \Lambda_{i}$ in the quasiWronskian $J_{n}$ are $2 \times 2$ matrices rather than a scalar function (cf. (2.10)). If the reader prefer the Wronskian determinants, just consider each matrix elements in the $2 \times 2$ matrix $J_{n}$ as the ratio of the Wronskian determinants (cf. (2.11)). If doing so, you will find that the analysis becomes much more complicated than the discussion of using quasideterminants.

Firstly, let us pick an $I \in\{1,2, \ldots, n\}$ and keep $L_{I}$ (and $\bar{L}_{I}$ ) to be finite (this is in fact equivalent to consider a comoving frame with the $I$-th soliton). If we take the asymptotic limit $r:=\left(\left(x^{0}\right)^{2}+\left(x^{1}\right)^{2}+\left(x^{2}\right)^{2}+\left(x^{3}\right)^{2}\right)^{1 / 2} \rightarrow \infty$ on $J_{n}$. Then for $i \neq I, \operatorname{Re} L_{i}\left(=\operatorname{Re} \bar{L}_{i}\right)$ goes to (i) positive infinity or (ii) negative infinity, that is, $\left|e^{L_{i}}\right|\left(=\left|e^{\bar{L}_{i}}\right|\right)$ goes to (i) positive 
infinity or (ii) zero. Now we can use (2.5) to eliminate common factors in each column $(i \neq I)$ of $J_{n}$ and take $r \rightarrow \infty$ to obtain the following asymptotic form:

$$
J_{n} \rightarrow\left|\begin{array}{cccccc}
C_{1} & \cdots & \theta_{I} & \cdots & C_{n} & 1 \\
C_{1} \Lambda_{1} & \cdots & \theta_{I} \Lambda_{I} & \cdots & C_{n} \Lambda_{n} & 0 \\
\vdots & & \vdots & & \vdots & \vdots \\
C_{1} \Lambda_{1}^{n} & \cdots & \theta_{I} \Lambda_{I}^{n} & \cdots & C_{n} \Lambda_{n}^{n} & 0
\end{array}\right| \text { where (i) } C_{i}=\left(\begin{array}{ll}
1 & 0 \\
0 & 1
\end{array}\right) \text { or (ii) }\left(\begin{array}{cc}
0 & 1 \\
-1 & 0
\end{array}\right)
$$

Next, our goal is to remove all the $C_{i}$ from the asymptotic form such that the remaining elements are just $I$-th column and constant matrices $\Lambda_{i}$, for $i \neq I$. The case (i) is trivial, while the case (ii) can be done by adjusting the diagonal terms of $\Lambda_{i}$ to get the commutation relation:

$$
C_{i} \Lambda_{i}^{k}=\left(\begin{array}{cc}
0 & 1 \\
-1 & 0
\end{array}\right)\left(\begin{array}{cc}
\lambda_{i}^{k} & 0 \\
0 & \mu_{i}^{k}
\end{array}\right)=\left(\begin{array}{cc}
\mu_{i}^{k} & 0 \\
0 & \lambda_{i}^{k}
\end{array}\right)\left(\begin{array}{cc}
0 & 1 \\
-1 & 0
\end{array}\right),
$$

and by using (2.5) to remove all the right common factors. The explicit result is

$$
\begin{aligned}
& J_{n} \rightarrow\left|\begin{array}{cccccc}
1 & \cdots & \theta_{I} & \cdots & 1 & 1 \\
\Lambda_{1}^{( \pm)} & \cdots & \theta_{I} \Lambda_{I} & \cdots & \Lambda_{n}^{( \pm)} & 0 \\
\vdots & & \vdots & & \vdots & \vdots \\
\Lambda_{1}^{( \pm) n} & \cdots & \theta_{I} \Lambda_{I}^{n} & \cdots & \Lambda_{n}^{( \pm) n} & 0
\end{array}\right|, \\
& \Lambda_{i, i \neq I}^{( \pm)}:=\left(\begin{array}{cc}
\lambda_{i}^{( \pm)} & 0 \\
0 & \lambda_{i}^{(\mp)}
\end{array}\right)=\left\{\begin{array}{cc}
\left(\begin{array}{cc}
\lambda_{i}^{(+)} & 0 \\
0 & \lambda_{i}^{(-)}
\end{array}\right)(\mathrm{i}) \operatorname{Re} L_{i} \rightarrow+\infty & \left(\left|e^{L_{i}}\right| \rightarrow \infty\right) \\
\left(\begin{array}{cc}
\lambda_{i}^{(-)} & 0 \\
0 & \lambda_{i}^{(+)}
\end{array}\right)(\mathrm{ii}) \operatorname{Re} L_{i} \rightarrow-\infty & \left(\left|e^{L_{i}}\right| \rightarrow 0\right)
\end{array}\right.
\end{aligned}
$$

where we introduce new notations: $\lambda_{i}^{(+)}:=\lambda_{i}, \lambda_{i}^{(-)}:=\mu_{i}$ to unify various cases of (i) and (ii) in the discussions and proofs of this subsection.

Now we need to show that the asymptotic form of $J_{n}$ is essentially the same as onesoliton solution (cf. (4.5) with $\theta=\theta_{I}$ ). Without loss of generality, we can consider the case of $I=1$ for convenience. The discussions of other cases are the same because of the permutation property of the quasideterminants (Propositon 2.1 (i)). By applying the noncommutative Jacobi identity (2.7) and (2.5) to the asymptotic form, $J_{n}$ can be represented as the product of three kind of quasideterminants defined as follows:

$$
J_{n} \rightarrow\left|\begin{array}{ccccc}
\theta_{1} & 1 & \cdots & 1 & 1 \\
\theta_{1} \Lambda_{1} & \Lambda_{2}^{( \pm)} & \cdots & \Lambda_{n}^{( \pm)} & 0 \\
\vdots & \vdots & & \vdots & \vdots \\
\theta_{1} \Lambda_{1}^{n-1} & \Lambda_{2}^{( \pm) n-1} & \cdots & \Lambda_{n}^{( \pm) n-1} & 0 \\
\theta_{1} \Lambda_{1}^{n} & \Lambda_{2}^{( \pm) n} & \cdots & \Lambda_{n}^{( \pm) n} & 0
\end{array}\right|=-Q_{n} \Lambda_{1} Q_{n}^{-1} D_{n}
$$


where

$$
Q_{n}:=\left|\begin{array}{cccc}
\theta_{1} & 1 & \cdots & 1 \\
\theta_{1} \Lambda_{1} & \Lambda_{2}^{( \pm)} & \cdots & \Lambda_{n}^{( \pm)} \\
\vdots & \vdots & & \vdots \\
\theta_{1} \Lambda_{1}^{n-2} & \Lambda_{2}^{( \pm) n-2} & \cdots & \Lambda_{n}^{( \pm) n-2} \\
\theta_{1} \Lambda_{1}^{n-1} & \Lambda_{2}^{( \pm) n-1} & \cdots & \Lambda_{n}^{( \pm) n-1}
\end{array}\right|, \quad D_{n}:=\left|\begin{array}{cccc}
0 & 1 & \cdots & 1 \\
0 & \Lambda_{2}^{( \pm)} & \cdots & \Lambda_{n}^{( \pm)} \\
\vdots & \vdots & & \vdots \\
0 & \Lambda_{2}^{( \pm) n-2} & \cdots & \Lambda_{n}^{( \pm) n-2} \\
1 & \Lambda_{2}^{( \pm) n-1} & \cdots & \Lambda_{n}^{( \pm) n-1}
\end{array}\right|^{-1} .
$$

In fact, $Q_{n}$ and $D_{n}$ can be expanded explicitly as the following (4.10) and (4.11), respectively. The proof is made by using mathematical induction. For $n \geq 2$ :

$$
\begin{aligned}
& Q_{n}=\left(\begin{array}{cc}
\left(\lambda_{1}-\lambda_{2}^{( \pm)}\right) \cdots\left(\lambda_{1}-\lambda_{n}^{( \pm)}\right) a_{1} e^{L_{1}} & \left(\mu_{1}-\lambda_{2}^{( \pm)}\right) \cdots\left(\mu_{1}-\lambda_{n}^{( \pm)}\right) b_{1} e^{-\bar{L}_{1}} \\
-\left(\lambda_{1}-\lambda_{2}^{(\mp)}\right) \cdots\left(\lambda_{1}-\lambda_{n}^{(\mp)}\right) \bar{b}_{1} e^{-L_{1}} & \left(\mu_{1}-\lambda_{2}^{(\mp)}\right) \cdots\left(\mu_{1}-\lambda_{n}^{(\mp)}\right) a_{1} e^{\bar{L}_{1}}
\end{array}\right), \\
& D_{n}=(-1)^{n-1} \Lambda_{n}^{( \pm)} \cdots \Lambda_{2}^{( \pm)} .
\end{aligned}
$$

For $n=2$, the statement is clearly true because we can easily check that

$$
Q_{2}=\left|\begin{array}{cc}
\theta_{1} & 1 \\
\theta_{1} \Lambda_{1} & \Lambda_{2}^{( \pm)}
\end{array}\right|=\theta_{1} \Lambda_{1}-\Lambda_{2}^{( \pm)} \theta_{1}, \quad D_{2}=\left|\begin{array}{cc}
0 & 1 \\
1 & \Lambda_{2}^{( \pm)}
\end{array}\right|^{-1}=-\Lambda_{2}^{( \pm)}
$$

by the Schur complements (2.4). Let us assume that the statement holds for some $n$. By using the noncommutative Jacobi identity (2.7) and (2.5), we have

$$
\begin{aligned}
& Q_{n+1}=\left|\begin{array}{cccc}
\theta_{1} \Lambda_{1} & \Lambda_{2}^{( \pm)} & \cdots & \Lambda_{n}^{( \pm)} \\
\theta_{1} \Lambda_{1}^{2} & \Lambda_{2}^{( \pm) 2} & \cdots & \Lambda_{n}^{( \pm) 2} \\
\vdots & \vdots & & \vdots \\
\theta_{1} \Lambda_{1}^{n} & \Lambda_{2}^{( \pm) n} & \cdots & \Lambda_{n}^{( \pm) n}
\end{array}\right| \\
& -\left|\begin{array}{ccc||ccc}
\Lambda_{2}^{( \pm)} & \cdots & \Lambda_{n+1}^{( \pm)} \\
\Lambda_{2}^{( \pm) 2} & \cdots & \Lambda_{n+1}^{( \pm) 2} \\
\vdots & & \vdots \\
\Lambda_{2}^{( \pm) n} & \cdots & \Lambda_{n+1}^{( \pm) n}
\end{array}\right|\left|\begin{array}{ccc}
1 & \cdots & 1 \\
\Lambda_{2}^{( \pm)} & \cdots & \Lambda_{n+1}^{( \pm)} \\
\vdots & & \vdots \\
\Lambda_{2}^{( \pm) n-1} & \cdots & \Lambda_{n+1}^{( \pm) n-1}
\end{array}\right|^{-1}\left|\begin{array}{cccc}
\theta_{1} & 1 & \cdots & 1 \\
\theta_{1} \Lambda_{1} & \Lambda_{2}^{( \pm)} & \cdots & \Lambda_{n}^{( \pm)} \\
\vdots & \vdots & & \vdots \\
\theta_{1} \Lambda_{1}^{n-1} & \Lambda_{2}^{( \pm) n-1} & \cdots & \Lambda_{n}^{( \pm) n-1}
\end{array}\right|
\end{aligned}
$$

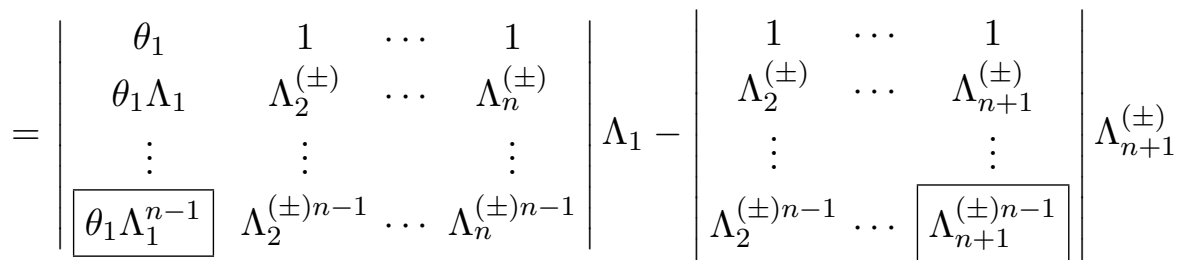

$$
\begin{aligned}
& \times\left|\begin{array}{ccc}
1 & \cdots & 1 \\
\Lambda_{2}^{( \pm)} & \cdots & \Lambda_{n+1}^{( \pm)} \\
\vdots & & \vdots \\
\Lambda_{2}^{( \pm) n-1} & \cdots & \Lambda_{n+1}^{( \pm) n-1}
\end{array}\right|^{-1}\left|\begin{array}{cccc}
\theta_{1} & 1 & \cdots & 1 \\
\theta_{1} \Lambda_{1} & \Lambda_{2}^{( \pm)} & \cdots & \Lambda_{n}^{( \pm)} \\
\vdots & \vdots & & \vdots \\
\theta_{1} \Lambda_{1}^{n-1} & \Lambda_{2}^{( \pm) n-1} & \cdots & \Lambda_{n}^{( \pm) n-1}
\end{array}\right|
\end{aligned}
$$


By using homological relation (2.8), we can expand the last two factors of $Q_{n+1}$ as

$$
\begin{aligned}
& \left|\begin{array}{ccc}
1 & \cdots & 1 \\
\Lambda_{2}^{( \pm)} & \cdots & \Lambda_{n+1}^{( \pm)} \\
\vdots & & \vdots \\
\Lambda_{2}^{( \pm) n-1} & \cdots & \Lambda_{n+1}^{( \pm) n-1}
\end{array}\right| \\
& =\left|\begin{array}{cccc}
1 & \cdots & 1 & 0 \\
\Lambda_{2}^{( \pm)} & \cdots & \Lambda_{n}^{( \pm)} & 0 \\
\vdots & & \vdots & 0 \\
\Lambda_{2}^{( \pm) n-1} & \cdots & \Lambda_{n}^{( \pm) n-1} & 1
\end{array}\right|\left|\begin{array}{ccc}
1 & \cdots & 1 \\
\Lambda_{2}^{( \pm)} & \cdots & \Lambda_{n+1}^{( \pm)} \\
\vdots & & \vdots \\
\Lambda_{2}^{( \pm) n-1} & \cdots & \Lambda_{n+1}^{( \pm) n-1}
\end{array}\right| \\
& \left|\begin{array}{cccc}
\theta_{1} & 1 & \cdots & 1 \\
\theta_{1} \Lambda_{1} & \Lambda_{2}^{( \pm)} & \cdots & \Lambda_{n}^{( \pm)} \\
\vdots & \vdots & & \vdots \\
\theta_{1} \Lambda_{1}^{n-1} & \Lambda_{2}^{( \pm) n-1} & \cdots & \Lambda_{n}^{( \pm) n-1}
\end{array}\right| \\
& =\left|\begin{array}{cccc}
0 & 1 & \cdots & 1 \\
0 & \Lambda_{2}^{( \pm)} & \cdots & \Lambda_{n}^{( \pm)} \\
0 & \vdots & & \vdots \\
1 & \Lambda_{2}^{( \pm) n-1} & \cdots & \Lambda_{n}^{( \pm) n-1}
\end{array}\right|\left|\begin{array}{cccc}
\theta_{1} & 1 & \cdots & 1 \\
\theta_{1} \Lambda_{1} & \Lambda_{2}^{( \pm)} & \cdots & \Lambda_{n}^{( \pm)} \\
\vdots & \vdots & & \vdots \\
\theta_{1} \Lambda_{1}^{n-1} & \Lambda_{2}^{( \pm) n-1} & \cdots & \Lambda_{n}^{( \pm) n-1}
\end{array}\right| .
\end{aligned}
$$

We note that the two quasideterminants involving 0 element are exactly equal to each other by Schur complements (2.4). Substituting the above equations into $Q_{n+1}$, and using the fact that $\left[\Lambda_{i}, \Lambda_{j}\right]=0$, we get

$$
\begin{aligned}
Q_{n+1} & =Q_{n} \Lambda_{1}-\Lambda_{n+1}^{( \pm)} Q_{n} \\
& =\left(\begin{array}{cc}
\left(\lambda_{1}-\lambda_{2}^{( \pm)}\right) \cdots\left(\lambda_{1}-\lambda_{n+1}^{( \pm)}\right) a_{1} e^{L_{1}} & \left(\mu_{1}-\lambda_{2}^{( \pm)}\right) \cdots\left(\mu_{1}-\lambda_{n+1}^{( \pm)}\right) b_{1} e^{-\bar{L}_{1}} \\
-\left(\lambda_{1}-\lambda_{2}^{(\mp)}\right) \cdots\left(\lambda_{1}-\lambda_{n+1}^{(\mp)}\right) \bar{b}_{1} e^{-L_{1}} & \left(\mu_{1}-\lambda_{2}^{(\mp)}\right) \cdots\left(\mu_{1}-\lambda_{n+1}^{(\mp)}\right) a_{1} e^{\bar{L}_{1}}
\end{array}\right) .
\end{aligned}
$$

As for $D_{n}$, we can use the noncommutative Jacobi identity (2.7) and the homological relation (2.8) to get

$$
\begin{aligned}
& D_{n}=-\left|\begin{array}{ccc}
1 & \cdots & 1 \\
\Lambda_{2}^{( \pm)} & \cdots & \Lambda_{n}^{( \pm)} \\
\vdots & & \vdots \\
\Lambda_{2}^{( \pm) n-2} & \cdots & \Lambda_{n}^{( \pm) n-2}
\end{array}\right| \Lambda_{n}^{( \pm)}\left|\begin{array}{ccc}
1 & \cdots & 1 \\
\Lambda_{2}^{( \pm)} & \cdots & \Lambda_{n}^{( \pm)} \\
\vdots & & \vdots \\
\Lambda_{2}^{( \pm) n-2} & \cdots & \Lambda_{n}^{( \pm) n-2}
\end{array}\right|^{-1} \\
& =-\left.\Lambda_{n}^{( \pm)}\left|\begin{array}{cccc}
1 & \cdots & 1 & 0 \\
\Lambda_{2}^{( \pm)} & \cdots & \Lambda_{n-1}^{( \pm)} & 0 \\
\vdots & & \vdots & 0 \\
\Lambda_{2}^{( \pm) n-2} & \cdots & \Lambda_{n-1}^{( \pm) n-2} & 1
\end{array}\right|\right|^{-1}
\end{aligned}
$$

By continuing the same process $n-1$ times, we can prove the statement (4.11). 
Now let us return back to the general situation that $I$ is not specified to $I=1$. So far, we have shown that the asymptotic form of $J_{n}$ can be expressed as (4.9) in terms of $Q_{n}$ of (4.10) and $D_{n}$ of (4.11). $D_{n}$ is a constant matrix and would not affect the result of action density. Hence in the original situation for finite $L_{I}$, the asymptotic form is essentially $J_{n} \sim Q_{n} \Lambda_{I} Q_{n}^{-1}$ which is in a very similar form of a one-soliton solution with $Q_{n}=\theta_{I}$ (cf. (4.7) and (4.10)). Under some conditions, for instance $\mu_{i}=\bar{\lambda}_{i}$, for all $i$ (not the unique choice), $Q_{n}$ can be simplified in a more concise form:

$$
Q_{n}=\left(\begin{array}{cc}
a_{I}^{\prime} e^{L_{I}} & b_{I}^{\prime} e^{-\bar{L}_{I}} \\
-\bar{b}_{I}^{\prime} e^{-L_{I}} & \bar{a}_{I}^{\prime} e^{\bar{L}_{I}}
\end{array}\right) .
$$

which is exactly in the form of one-soliton solution now. This is what we want to show here. The only difference between $Q_{n}$ and $\theta_{I}$ is the constants $a_{I}^{\prime}, b_{I}^{\prime}$ and $a_{I}, b_{I}$ which relate to the position of the principal peak of the action density (cf. (4.6)). This difference gives rise to additional position shift, called the phase shift. Now we can conclude that the action density calculated from the asymptotic form of $J_{n}$ and $Q_{n}$ is almost the same as (4.6) with $\theta=\theta_{I}$. In other word, if we consider the comoving frame with $I$-th one-soliton, the asymptotic multi-soliton inherits almost the same features from the $I$-th one-soliton except for the phase shift factor.

It is time for us to calculate the phase shift factor $\Delta_{I, n}$ explicitly. Firstly, we take the Ultrahyperbolic space $\mathbb{U}$ for example, (4.12) is obviously satisfied because $\mu_{i}=\bar{\lambda}_{i}$, for all $i$ (cf. table 1). Then the action density becomes

$$
\operatorname{Tr} F_{\mu \nu} F^{\mu \nu}=8\left[\left(\alpha_{I} \bar{\beta}_{I}-\bar{\alpha}_{I} \beta_{I}\right)\left(\lambda_{I}-\bar{\lambda}_{I}\right)\right]^{2}\left(2 \operatorname{sech}^{2} X_{I}-3 \operatorname{sech}^{4} X_{I}\right),
$$

where $X_{I}=L_{I}+\bar{L}_{I}+\log \left|a_{I}^{\prime} / b_{I}^{\prime}\right|=L_{I}+\bar{L}_{I}+\log \left|a_{I} / b_{I}\right|+\Delta_{I, n}^{\mathbb{U}}$. The phase shift factor can be calculated by (4.4). The result is exactly real-valued

$$
\Delta_{I, n}^{\mathbb{U}}=\sum_{k=1(\neq I)}^{n} \varepsilon_{k} \log \left|\frac{\lambda_{I}-\lambda_{k}}{\lambda_{I}-\bar{\lambda}_{k}}\right|,
$$

where $\varepsilon_{k}=+1$ for case (i) and $\varepsilon_{k}=-1$ for case (ii).

For the Euclidean signature, we can use (4.4) and the reality condition $\mu_{i}=-1 / \bar{\lambda}_{i}$, for all $i$ (cf. table 1 ) to calculate the phase shift, the result is also real-valued:

$$
\Delta_{I, n}^{\mathbb{E}}=\sum_{k=1(\neq I)}^{n} \varepsilon_{k} \log \left|\frac{\lambda_{I}-\lambda_{k}}{1+\lambda_{I} \bar{\lambda}_{k}}\right| .
$$

For the Minkowski signature, if we don't impose additional condition on $\lambda_{i}$ and $\mu_{i}$ as that in one-soliton case (cf. table 1), the phase shift factor is complex-valued in general. This shortcoming can be solved immediately, for example, we can take $\mu_{i}=\bar{\lambda}_{i}$ or $\mu_{i}=-1 / \bar{\lambda}_{i}$ like that in $\mathbb{U}$ and $\mathbb{E}$, respectively.

In summary, the asymptotic behavior of the solutions (4.7) can be interpreted as $n$ intersecting soliton walls with phase shifts in the scattering process. It is a well-known property for the KP multi-solitons, but a new insight for anti-self-dual Yang-Mills multi-solitons. 


\section{Unitarity of the multi-soliton solutions in $\mathbb{U}$}

In this section, we discuss the unitarity of multi-soliton solutions for physical purpose. Recall that in table 1, we get unitary one-soliton solutions (soliton walls) successfully on the Ultrahyperbolic space $\mathbb{U}$ and hence they could be realized as branes of three-dimensions in the $\mathrm{N}=2$ string theory as commented in introduction. Therefore the multi-soliton solutions (the intersecting soliton walls) in unitary gauge group case can be interpreted as $n$ intersecting branes in the $\mathrm{N}=2$ string theory.

To prove the unitarity of $n$ intersecting soliton walls, it suffices to verify the hermiticity of gauge fields. By imposing the real slice condition (cf. table 1) of Ultrahyperbolic space $\mathbb{U}$ on the gauge fields (3.2), we obtain the Ultrahyperbolic version of gauge fields:

$$
A_{0}=-A_{2}=\frac{1}{2}\left(\partial_{2} J \cdot J^{-1}-\partial_{0} J \cdot J^{-1}\right), \quad A_{1}=-A_{3}=\frac{1}{2}\left(\partial_{3} J \cdot J^{-1}-\partial_{1} J \cdot J^{-1}\right) .
$$

We will soon see that $J$-matrix is constant multiple of the special unitary matrix, and hence all the gauge fields $A_{\mu}(\mu=0,1,2,3)$ are anti-hermitian and traceless.

In order to discuss it, let us define a class of $2 \times 2$ matrices. If $P \in \mathrm{GL}(2, \mathbb{C})$ has the following form,

$$
P=\left(\begin{array}{cc}
\alpha & \bar{\beta} \\
-\beta & \bar{\alpha}
\end{array}\right), \quad \alpha, \beta \in \mathbb{C},
$$

then we call $P$ the pre-SU(2) matrix in this paper. We can easily find that the pre-SU(2) matrix $P$ can be represented by the product of det $P$ and a SU(2) matrix and hence satisfies $P^{\dagger} P=P P^{\dagger}=\operatorname{det} P$. Furthermore, if two $2 \times 2$ matrices $P$ and $Q$ are pre-SU(2), then $P \pm Q, P Q$, and $P^{-1}, Q^{-1}$ are also pre-SU(2).

By the iterative representions of quasideterminants (cf. (2.3)), the $n$-soliton solution $J_{n}$ in (4.7) is found to be a polynomial of $2 \times 2$ matrices $\theta_{i}, \theta_{i}^{-1}, \Lambda_{i}, \Lambda_{i}^{-1}$ which are all pre-SU(2). Therefore $J_{n}$ is also pre-SU(2) satisfying $J_{n}^{\dagger} J_{n}=J_{n} J_{n}^{\dagger}=\operatorname{det} J_{n}$. On the other hand, we can prove that $\operatorname{det} J_{n}=\prod_{i=1}^{n}\left|\lambda_{i}\right|^{2}$ by the recursion relation: $\operatorname{det} J_{n}=$ $\operatorname{det}\left(\Lambda_{n}\right) \operatorname{det} J_{n-1}=\left|\lambda_{n}\right|^{2} \operatorname{det} J_{n-1}$, $\operatorname{det} J_{0}=1$ which comes from the explicit form (3.5) of the Darboux transformation of $J_{k}(k=1, \cdots, n)$.

Now we can prove the gauge fields (5.1) are anti-hermitian and traceless because of $\left(\partial_{\mu} J_{n}\right) J_{n}^{-1}=\left(\partial_{\mu} \widetilde{J}_{n}\right) \widetilde{J}_{n}^{-1}$ where $\widetilde{J}_{n}$ is a $\mathrm{SU}(2)$ matrix defined by $\widetilde{J}_{n}:=R^{-1 / 2} J_{n}$ in which $R:=\prod_{i=1}^{n}\left|\lambda_{i}\right|^{2}$. Therefore we can conclude that the $n$ intersecting soliton walls can be embedded into $G=\mathrm{SU}(2)$ gauge theory on the Ultrahyperbolic space $\mathbb{U}$.

\section{Conclusion and discussion}

In this paper, we discussed multi-soliton dynamics of anti-self-dual Yang-Mills equations by analyzing the action density in the asymptotic region. By considering a comoving frame with the $I$-th soliton, we proved that the entire multi-soliton distribution is asymptotically equal to the $I$-th one-soliton distribution except for a phase shift, and we also calculated the phase shifts explicitly. Therefore, our results can be interpreted as intersecting soliton 
walls with phase shifts in the scattering process. It is surprising that this behavior is quite similar to the case of KP soliton scattering [28, 30], and it suggests the viability of Sato's formulation for anti-self-dual Yang-Mills equations. Furthermore, we proved that in the Ultrahyperbolic space $\mathbb{U}$, the $J$-matrix is unitary. Hence the multi-soliton solutions can be embedded into $\mathrm{SU}(2)$ gauge theory. This implies that there would exist intersecting branes of three-dimensions in the $\mathrm{N}=2$ string theory.

In our current work, we focused on the pure scattering process of soliton walls and excluded the case of resonance processes. The resonance processes describe soliton wall reconnections. Actually, the classification of all possible soliton wall distributions (containing the resonance processes) could be put into practice by adjusting the parameters of (4.7) properly (cf. [30]) or by following a similar strategy of Kodama and Williams [22, 23] from the viewpoint of positive Grassmannians.

On the other hand, the quasi-Wronskian solutions here might have a similar representations of Maya diagrams or tau functions in Sato's theory, and relate to generalized Schur functions (cf. [7, 37]). Perhaps after a more comprehensive study from this perspective, the realization of Sato's theory for the anti-self-dual Yang-Mills equation version might be achieved even though it is still a challenging problem (cf. [38]).

The extension of integrable systems to noncommutative space-time is not our aim in this paper, but it is still a potentially interesting topic (for reviews see, e.g. [16, 17, 24]). In the previous work [10], we showed that on the Euclidean space $\mathbb{E}$, noncommutative multi-soliton solutions of anti-self-dual Yang-Mills equations is equivalent to the commutative ones in the asymptotic region. By quite similar arguments, we can make the same conclusion on the Ultrahyperbolic space $\mathbb{U}$. This means that on $\mathbb{U}$, the behavior of threedimensional branes (soliton walls) in the asymptotic region are not affected by the background $B$-field. On the other hand, the soliton equations of lower-dimensional integrable systems can be derived from the anti-self-dual Yang-Mills equations by suitable reduction procedure even when the space-time coordinates are noncommutative [14]. Therefore, the techniques presented in this paper could be applied to the lower-dimensional soliton equations even in noncommutative space-time because quasideterminants are especially suitable for the description of noncommutative integrable systems (e.g. $[6,11,12,36])$. Furthermore, the asymptotic behaviors of noncommutative multi-soliton solutions are proved to be the same as the commutative ones in lower-dimensional integrable systems (e.g. [4, 15, 18, 33]), but the physical interpretation are still uncertain.

\section{Acknowledgments}

The work of MH was supported by Grant-in-Aid for Scientific Research (\#16K05318). The work of SCH is supported by the scholarship of Japan-Taiwan Exchange Association.

Open Access. This article is distributed under the terms of the Creative Commons Attribution License (CC-BY 4.0), which permits any use, distribution and reproduction in any medium, provided the original author(s) and source are credited. 


\section{References}

[1] L.-L. Chau, J.C. Shaw and H.C. Yen, $N$ soliton type solutions of SU(2) SDYM equations in various spaces and their Backlund transformations, J. Phys. A 27 (1994) 7131 [INSPIRE].

[2] E. Corrigan, D.B. Fairlie, R.G. Yates and P. Goddard, The construction of selfdual solutions to SU(2) gauge theory, Commun. Math. Phys. 58 (1978) 223 [INSPIRE].

[3] H.J. de Vega, Nonlinear multiplane wave solutions of selfdual Yang-Mills theory, Commun. Math. Phys. 116 (1988) 659 [INSPIRE].

[4] A. Dimakis and F. Mueller-Hoissen, Noncommutative Korteweg-de Vries equation, Phys. Lett. A 278 (2000) 139 [hep-th/0007074] [INSPIRE].

[5] M. Dunajski, Solitons, instantons, and twistors, Oxford University Press, Oxford U.K. (2010).

[6] P. Etingof, I. Gelfand and V. Retakh, Factorization of differential operators, quasideterminants, and nonabelian Toda field equations, Math. Res. Lett. 4 (1997) 413 [q-alg/9701008].

[7] I.M. Gelfand, D. Krob, A. Lascoux, B. Leclerc, V.S. Retakh and J.-Y. Thibon, Noncommutative symmetric functions, hep-th/9407124 [INSPIRE].

[8] I. Gelfand, S. Gelfand, V. Retakh and R. Wilson, Quasideterminants, Adv. Math. 193 (2005) 56 [math.QA/0208146].

[9] I. Gelfand and V. Retakh, Determinants of matrices over noncommutative rings, Funct. Anal. Appl. 25 (1991) 91 [Funct. Anal. Appl. 2 (1992) 1].

[10] C.R. Gilson, M. Hamanaka, S.-C. Huang and J.J.C. Nimmo, Soliton solutions of noncommutative anti-self-dual Yang-Mills equations, J. Phys. A 53 (2020) 404002 [arXiv:2004.01718] [INSPIRE].

[11] C.R. Gilson, M. Hamanaka and J.J.C. Nimmo, Backlund transformations and the Atiyah-Ward ansatz for noncommutative anti-self-dual Yang-Mills equations, Proc. Roy. Soc. Lond. A 465 (2009) 2613 [arXiv:0812.1222] [INSPIRE].

[12] C.R. Gilson and J.J.C. Nimmo, On a direct approach to quasideterminant solutions of a noncommutative KP equation, J. Phys. A 40 (2007) 3839 [nlin/0701027].

[13] D. Gluck, Y. Oz and T. Sakai, D-branes in $N=2$ strings, JHEP 08 (2003) 055 [hep-th/0306112] [InSPIRE].

[14] M. Hamanaka, Noncommutative Ward's conjecture and integrable systems, Nucl. Phys. B 741 (2006) 368 [hep-th/0601209] [INSPIRE].

[15] M. Hamanaka, Notes on exact multi-soliton solutions of noncommutative integrable hierarchies, JHEP 02 (2007) 094 [hep-th/0610006] [INSPIRE].

[16] M. Hamanaka, Noncommutative integrable systems and quasideterminants, AIP Conf. Proc. 1212 (2010) 122 [arXiv: 1012.6043] [INSPIRE].

[17] M. Hamanaka, Noncommutative solitons and quasideterminants, Phys. Scripta 89 (2014) 038006 [arXiv: 1101.0005] [INSPIRE].

[18] M. Hamanaka and H. Okabe, Soliton scattering in noncommutative spaces, Theor. Math. Phys. 197 (2018) 1451 [Teor. Mat. Fiz. 197 (2018) 68] [arXiv:1806.05188] [INSPIRE]. 
[19] M. Hamanaka and S.-C. Huang, New soliton solutions of anti-self-dual Yang-Mills equations, JHEP 10 (2020) 101 [arXiv:2004.09248] [INSPIRE].

[20] M. Hamanaka and K. Toda, Towards noncommutative integrable systems, Phys. Lett. A 316 (2003) 77 [hep-th/0211148] [INSPIRE].

[21] S.C. Huang, On soliton solutions of the anti-self-dual Yang-Mills equations from the perspective of integrable systems, arXiv:2112.10702.

[22] Y. Kodama, KP solitons and the Grassmannians, Springer, Germany (2017).

[23] Y. Kodama and L. Williams, KP solitons and total positivity for the Grassmannian, Inv. Math. 198 (2014) 637 [arXiv: 1106. 0023] [INSPIRE].

[24] O. Lechtenfeld, Noncommutative solitons, hep-th/0605034 [INSPIRE].

[25] N. Marcus, The $N=2$ open string, Nucl. Phys. B 387 (1992) 263 [hep-th/9207024] [INSPIRE].

[26] L.J. Mason, Global anti-self-dual Yang-Mills fields in split signature and their scattering, math-ph/0505039 [INSPIRE].

[27] L.J. Mason and N.M. Woodhouse, Integrability, self-duality, and twistor theory, Oxford University Press, U.K. (1996).

[28] V.B. Matveev and M.A. Salle, Darboux transformations and solitons, Springer, Germany (1991).

[29] J.J.C. Nimmo, C.R. Gilson and Y. Ohta, Applications of Darboux transformations to the selfdual Yang-Mills equations, Theor. Math. Phys. 122 (2000) 239 [Teor. Mat. Fiz. 122 (2000) 284] [INSPIRE].

[30] K. Ohkuma and M. Wadati, The Kadomtsev-Petviashvili equation: the trace method and the soliton resonances, J. Phys. Soc. Jap. 52 (1983) 749.

[31] H. Ooguri and C. Vafa, Geometry of N=2 strings, Nucl. Phys. B 361 (1991) 469 [inSPIRE].

[32] H. Ooguri and C. Vafa, $N=2$ heterotic strings, Nucl. Phys. B 367 (1991) 83 [INSPIRE].

[33] L.D. Paniak, Exact noncommutative KP and KdV multisolitons, hep-th/0105185 [INSPIRE].

[34] R. Penrose and W. Rindler, Spinors and space-time. Volume 1, Cambridge University Press, Cambridge U.K. (1984).

[35] R. Penrose and W. Rindler, Spinors and space-time. Volume 2, Cambridge University Press, Cambridge U.K. (1986).

[36] V. Retakh and V. Rubtsov, Noncommutative Toda chains, Hankel quasideterminants and Painlevé II equation, J. Phys. A 43 (2010) 505204 [arXiv: 1007.4168].

[37] N. Sasa, Y. Ohta and J. Matsukidaira, Bilinear form approach to the self-dual Yang-Mills equation and integrable system in (2+1)-dimension, J. Phys. Soc. Jap. 67 (1998) 83.

[38] M. Sato, Lectures by Sato Mikio (in Japanese), RIMS lecture note (1989).

[39] M. Sato, Soliton equations as dynamical systems on a infinite dimensional Grassmann manifolds (random systems and dynamical systems), RIMS Kokyuroku 439 (1981) 30.

[40] M. Sato and Y. Sato, Soliton equations as dynamical systems on infinite dimensional Grassmann manifold, Noth Holland, The Netherlands (1983). 
[41] K. Takasaki, A new approach to the selfdual Yang-Mills equations, Commun. Math. Phys. 94 (1984) 35 [INSPIRE].

[42] R.S. Ward, Integrable and solvable systems, and relations among them, Phil. Trans. Roy. Soc. Lond. A 315 (1985) 451.

[43] R.S. Ward and R. Wells, Twistor geometry and field theory, Cambridge University Press, Cambridge U.K. (1990). 